\title{
Spin and orbital fluctuations in non-equilibrium transport through quantum dots: A renormalisation-group analysis
}

\author{
S. Y. Müller, ${ }^{1}$ V. Koerting,,$^{2,3}$ D. Schuricht,${ }^{1}$ and S. Andergassen ${ }^{1}$ \\ ${ }^{1}$ Institut für Theorie der statistischen Physik and JARA-Fundamentals of \\ Future Information Technology, RWTH Aachen, 52056 Aachen, Germany \\ ${ }^{2}$ Nano-Science Center, Niels Bohr Institute, Universitetsparken 5, 2100 Copenhagen, Denmark \\ ${ }^{3}$ Niels Bohr International Academy, Niels Bohr Institute, Blegdamsvej 17, 2100 Copenhagen, Denmark
}

(Dated: June 15, 2018)

\begin{abstract}
We study non-equilibrium current and occupation probabilities of a two-orbital quantum dot. The couplings to the leads are allowed to be asymmetric and orbital dependent as it is generically the case in transport experiments on molecules and nanowires. Starting from a two-orbital Anderson model, we perform a generalised Schrieffer-Wolff transformation to derive an effective Kondo model. This generates an orbital potential scattering contribution which is of the same order as the spin exchange interaction. In a first perturbative analysis we identify a regime of negative differential conductance and a cascade resonance in the presence of an external magnetic field, which both originate from the non-equilibrium occupation of the orbitals. We then study the logarithmic enhancement of these signatures by means of a renormalisation-group treatment. We find that the orbital potential scattering qualitatively changes the renormalisation of the spin exchange couplings and strongly affects the differential conductance for asymmetric couplings.

PACS numbers: 05.60.Gg, 73.63.Kv, 05.10.Cc
\end{abstract}

\section{INTRODUCTION}

Over the past decade the fast progress in nanofabrication allowed the observation of single electron tunneling as well as Coulomb blockade, cotunneling and Kondo signatures in quantum dot devices. Initially these dots were gate-controlled isolated islands in two-dimensional electron gases like GaAs [1]. Recently a new trend has evolved towards studying molecules in gated junctions. Starting from small molecules [2, 3] and bucky balls [4] also carbon nanotubes [5, 6] and InAs nanowires were studied [7]. In contrast to gate-defined quantum dots these molecules and nanowires possess a rich spectrum of internal degrees of freedom, which significantly influence non-equilibrium transport through these systems. In particular, the overlaps of the orbitals of a molecule with the states in the leads vary significantly, which results in asymmetric and orbital-dependent tunneling amplitudes.

Parallel to the experimental progress there have been numerous theoretical studies on non-equilibrium transport through nanostructures [8]. One of the main challenges consists in the new energy scale introduced by the bias voltage, which is in general of the same order as the internal energy scales of the system. The understanding of non-equilibrium phenomena in the presence of strong correlations represents an outstanding task in condensed matter physics which requires the development of powerful theoretical tools. Recent advances include various renormalisation group (RG) methods 9-11] introduced for the study of quantum dots mainly dominated by spin fluctuations.

Motivated by the experimental developments [2 7] we will study a quantum dot possessing multiple orbitals and asymmetric tunneling amplitudes. A previous per- turbative study [12] showed that such systems generically exhibit interesting non-equilibrium features like the socalled cascade effect and the appearance of a negative differential conductance (NDC). Our main focus is the analysis of the logarithmic enhancements of these features due to the Kondo effect and the interplay of spin and orbital degrees of freedom. Even without spin-orbit interaction the renormalisation of spin and orbital scattering processes can influence each other in multi-orbital quantum dots. The minimal model to observe these features is provided by a two-orbital quantum dot with finite orbital splitting, an external magnetic field, and differing tunneling amplitudes (see fig. 1).

We consider the Coulomb blockade region with a single electron on the dot. In this situation the orbital or spin degree of freedom can be changed by elastic or inelastic cotunneling processes. Using a Schrieffer-Wolff (SW) transformation [13] we derive a Kondo spin ex-

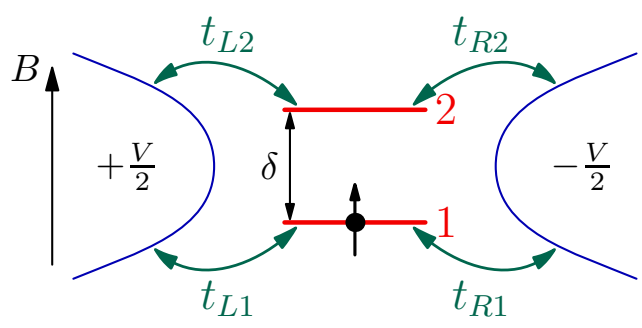

FIG. 1: (Colour online) Sketch of the two-orbital Anderson model. The energies of the two orbitals are split by $\delta$. The orbitals are coupled to free fermionic leads held at different chemical potentials $\pm V / 2$. The system is assumed to be in the regime of single occupancy and the dot orbitals are subject to an external magnetic field $B$. 
change as well as an orbital potential scattering (PS) interaction, which have the same strength and therefore must be treated on an equal footing. The orbital PS term includes two processes, one involving a change of the orbital index and one describing transport via the empty orbital.

We first study the orbital occupations and the differential conductance to second order in perturbation theory $(\mathrm{PT})$, which provides a reliable and comprehensive picture of the considered non-equilibrium physics. In the main part of the paper we perform an RG analysis with frequency-dependent couplings [9] and study the effect of the orbital PS. This method was recently used to study cotunneling transport through carbon nanotubes modelled by a two-orbital Anderson dot with two electrons [6]. While for this situation orbital PS contributions are expected to play an important role, the impact of an empty orbital remains to be assessed. Furthermore, transport through a double quantum dot coupled to four different leads was investigated in ref. [14]. In this work NDC with respect to one pair of the leads was observed and a detailed RG analysis of the transport properties as a function of the orbital splitting was performed.

In our set-up (see fig. 1) we study the dependence of the cascade resonance and the NDC on both the coupling asymmetry and the external magnetic field. By including the logarithmic corrections to the inelastic cotunneling processes we further show that the interplay between spin and orbital scattering is most pronounced for strong asymmetric couplings to the reservoirs. The detected enhancement of the NDC reveals the importance of Kondo correlations for the appearance of this effect, whose signatures can be probed in transport experiments [2].

\section{MODEL AND SW TRANSFORMATION}

We start from a two-orbital Anderson model sketched in fig. 1. The Hamiltonian is given by $H=H_{\text {leads }}+$ $H_{\text {dot }}+H_{\text {int }}$. In second quantisation the fermionic leads are described by $H_{\text {leads }}=\sum_{\alpha k \sigma}\left(\varepsilon_{\alpha k \sigma}-\mu_{\alpha}\right) c_{\alpha k \sigma}^{\dagger} c_{\alpha k \sigma}$ with $\alpha=L, R$, the chemical potentials $\mu_{\alpha}= \pm \mathrm{eV} / 2$ and the density of states $N(\omega)=N_{0} \Theta\left(D_{0}-|\omega|\right), N_{0}=1 /\left(2 D_{0}\right)$. The dot Hamiltonian reads

$$
H_{\mathrm{dot}}=\sum_{a s} \varepsilon_{a s} d_{a s}^{\dagger} d_{a s}+\frac{U}{2} \sum_{a s} d_{a s}^{\dagger} d_{a s}\left(\sum_{b s^{\prime}} d_{b s^{\prime}}^{\dagger} d_{b s^{\prime}}-1\right)
$$

with the orbital index $a=1,2$, the spin index $s= \pm 1$, and an orbital-independent local Coulomb interaction. The energies of the orbitals are $\varepsilon_{1 s}=\varepsilon_{1}-\frac{1}{2} s g \mu_{B} B$ and $\varepsilon_{2 s}=\varepsilon_{1 s}+\delta$ with an external magnetic field $B$. We stress that the orbital splitting $\delta$ is assumed to be finite. The orbitals are coupled to the leads by $H_{\mathrm{int}}=$ $\sum_{\alpha a \sigma}\left(t_{\alpha a} c_{\alpha \sigma}^{\dagger} d_{a \sigma}+\right.$ h.c. $)$ with spin-independent hopping amplitudes $t_{\alpha a}$ and $c_{\alpha \sigma}^{\dagger}=\sum_{k} c_{\alpha k \sigma}^{\dagger}$.

In the following we will consider the regime with exactly one electron on the dot, i.e. $\sum_{a s} n_{a s}=1$ with $n_{a s}=d_{a s}^{\dagger} d_{a s}$. We use a generalised SW transformation to derive an effective Kondo model $H_{\mathrm{K}}+H_{\mathrm{PS}}$ in the limit $\delta \ll U$. We find the conventional Kondo spin exchange interaction in its two-orbital form

$$
H_{\mathrm{K}}=\sum_{\alpha \beta} \sum_{a b} J_{b a}^{\beta \alpha} \vec{S}_{b a} \cdot \vec{s}_{\beta \alpha}
$$

with $J_{b a}^{\beta \alpha}=2 t_{\beta a} t_{\alpha b}^{*}\left(1 /\left(\varepsilon_{b}+U\right)-1 / \varepsilon_{a}\right), \quad \vec{S}_{b a}=$ $\frac{1}{2} \sum_{s s^{\prime}} d_{b s}^{\dagger} \vec{\tau}_{s s^{\prime}} d_{a s^{\prime}}$, and $\vec{s}_{\beta \alpha}=\frac{1}{2} \sum_{\sigma \sigma^{\prime}} c_{\beta \sigma}^{\dagger} \vec{\tau}_{\sigma \sigma^{\prime}} c_{\alpha \sigma^{\prime}}$. In an experimental set-up the overlaps between the states on the dot and in the leads will strongly depend on the lead and orbital indices, thus leading to a strong asymmetry as well as orbital dependence of the hopping amplitudes $t_{\alpha a}$ and the Kondo couplings $J_{b a}^{\beta \alpha}$. In addition to (2) the SW transformation generates a spin-independent orbital PS term

$$
H_{\mathrm{PS}}=\frac{1}{4} \sum_{\alpha \beta \sigma} c_{\beta \sigma}^{\dagger} c_{\alpha \sigma} \sum_{a s}\left(J_{\bar{a} a}^{\beta \alpha} d_{\bar{a} s}^{\dagger} d_{a s}-J_{\bar{a} \bar{a}}^{\beta \alpha} d_{a s}^{\dagger} d_{a s}\right),
$$

where $\bar{a}=2(1)$ if $a=1(2)$. The second term $\propto J_{\bar{a} \bar{a}}^{\beta \alpha}$ describes cotunneling transport across the empty orbital, e.g. orbital 2 if orbital 1 is occupied. The first term $\propto J_{\bar{a} a}^{\beta \alpha}$ is a spin-conserving process which changes the orbital state of the dot; we therefore expect a significant influence on the current at voltages $V \sim \delta$. We emphasise that (3) is of the same order as the Kondo interaction (2) and hence cannot be neglected. In particular, we show below that the orbital PS term qualitatively changes the RG flow of the Kondo couplings. In order to distinguish the Kondo and orbital PS contributions we will denote the couplings of the latter by $P^{0}\left(\equiv J_{\bar{a} \bar{a}}^{\beta \alpha}\right)$ and $P^{x}\left(\equiv J_{\bar{a} a}^{\beta \alpha}\right)$ in the following. Furthermore, we study the system at the particle-hole symmetric point, i.e. $\varepsilon_{1}=-U / 2-\delta / 2$. In this case the conventional single-orbital PS contribution $\left(\propto 1 /\left(\varepsilon_{a}+U\right)+1 / \varepsilon_{a}\right)$ can be neglected $(\delta \ll U$ has already been assumed in the SW transformation).

To simplify the notations we introduce a generalised vertex $\mathcal{V}$ via

$$
H_{\mathrm{K}}+H_{\mathrm{PS}}=\sum_{\alpha \beta a b} \sum_{s s^{\prime} \sigma \sigma^{\prime}} \mathcal{V}_{b s^{\prime} ; a s}^{\beta \sigma^{\prime} ; \alpha \sigma} d_{b s^{\prime}}^{\dagger} d_{a s} c_{\beta \sigma^{\prime}}^{\dagger} c_{\alpha \sigma},
$$

where

$$
\begin{aligned}
\mathcal{V}_{b s^{\prime}, a s}^{\beta \sigma^{\prime}, \alpha \sigma} & =\frac{1}{4} \tau_{\sigma \sigma^{\prime}}^{z} \tau_{s s^{\prime}}^{z}\left(J_{s}^{z}\right)_{b a}^{\beta \alpha}+\frac{1}{8}\left(\tau_{\sigma \sigma^{\prime}}^{+} \tau_{s s^{\prime}}^{-}+\tau_{\sigma \sigma^{\prime}}^{-} \tau_{s s^{\prime}}^{+}\right)\left(J_{s}^{\perp}\right)_{b a}^{\beta \alpha} \\
& +\frac{1}{4} \delta_{\sigma \sigma^{\prime}} \delta_{s s^{\prime}} \tau_{a b}^{x}\left(P_{s}^{x}\right)_{\bar{a} a}^{\beta \alpha}-\frac{1}{4} \delta_{\sigma \sigma^{\prime}} \delta_{s s^{\prime}} \delta_{a b}\left(P_{s}^{0}\right)_{\bar{a} \bar{a}}^{\beta \alpha} .
\end{aligned}
$$

Initially both interaction terms (2) and (3) are rotational symmetric in spin space, i.e. $J^{z}=J^{\perp}=P^{0}=P^{x}$ are independent of the spin index $s$ on the dot. As we will see below, the RG flow leads to a breaking of this symmetry.

Furthermore, from now on we use the Einstein summation convention and parametrise the couplings as $J_{b a}^{\beta \alpha}=$ $r_{\alpha b} r_{\beta a} J_{0}$ with $r_{\alpha b}=t_{\alpha b} / t_{L 1}$ and $t_{L 1}=t_{L 2}=1, t_{R 1}=r$, $t_{R 2}=1 / r$. We use $\delta$ as unit of energy $(\delta \equiv 1), r$ as asymmetry parameter and set $\hbar=k_{B}=e=g \mu_{B}=1$. 


\section{PT AND RG EQUATIONS}

We first study the system using second order PT. The current is computed using a rate-equation approach (see e.g. ref. 11]). Following ref. [15] we use a pseudofermion representation [16] for the Kondo spins; for details of the calculation we refer to ref. [17]. We define the dot Green's function, $G_{a s}\left(\tau, \tau^{\prime}\right)=-i\left\langle T_{C} d_{a s}(\tau) d_{a s}^{\dagger}\left(\tau^{\prime}\right)\right\rangle$, where $T_{C}$ is the contour ordering of the times $\tau, \tau^{\prime}$ on the Keldysh contour. For the lesser Green's function we assume $G_{a s}^{<}(\omega)=i n_{a s} A_{a s}(\omega)$, where $A_{a s}(\omega)$ denotes the non-interacting spectral function given by a $\delta$-function at $\varepsilon_{a s}$. The stationary occupation of the state as is determined by the rate equation

$$
\sum_{b s^{\prime}}\left(\Gamma_{a s \rightarrow b s^{\prime}} n_{a s}-\Gamma_{b s^{\prime} \rightarrow a s} n_{b s^{\prime}}\right)=0 .
$$

The rates are defined via the retarded self energy as $\sum_{b s^{\prime}} \Gamma_{a s \rightarrow b s^{\prime}} \equiv \Gamma_{a s}\left(\varepsilon_{a s}\right)=-2 \operatorname{Im}\left[\Sigma_{a s}^{R}\left(\varepsilon_{a s}\right)\right]$. In second order PT (Fermi's golden rule) we find

$$
\Gamma_{a s}\left(\varepsilon_{a s}\right)=2 \pi\left|N_{0} \mathcal{V}_{a s ; b s^{\prime}}^{\beta \sigma^{\prime} ; \alpha \sigma}\right|^{2} w\left(\mu_{\alpha}-\mu_{\beta}+\varepsilon_{b s^{\prime}}-\varepsilon_{a s}\right) .
$$

Here $w(x)=x n_{B}(x)$, with the finite-temperature Bose function $n_{B}(x)$, gives the energy window allowed for transitions. We note that the self-consistency equations (6) have to be solved under the constraint $\sum_{a s} n_{a s}=1$. Results of the perturbative treatment of the occupation numbers and the differential conductance are shown in figs. 3 and 4 .

In order to include Kondo correlations we use a nonequilibrium poor man's scaling approach with frequencydependent coupling functions [9]. The underlying approximations are devised for $\max (V, T, B) \gg T_{K}$ providing a cutoff for the flow to strong coupling. We start with the representation (4). During the RG flow the vertex $\mathcal{V}$ acquires a dependence on the energies of the incoming and outgoing lead electrons as well as the involved dot states. Approximating the latter by their respective resonance energies $\varepsilon_{a s}$ and using energy conservation we deduce that $\mathcal{V}$ will depend on the frequency $\omega$ of the incoming electron only. Thus during the RG flow we can use the parametrisation (5) with $\omega$-dependent couplings $J^{z}, J^{\perp}, P^{0}$ and $P^{x}$. Explicitly, the RG equation for the vertex reads

$$
\begin{aligned}
& \frac{\partial}{\partial \ln D} \mathcal{V}_{b s^{\prime}, a s}^{\beta \sigma^{\prime}, \alpha \sigma}(\omega)= \\
& \Theta_{\omega+\varepsilon_{a s}-\varepsilon_{c s^{\prime \prime}}-\mu_{\gamma}} \mathcal{V}_{b s^{\prime}, c s^{\prime \prime}}^{\beta \sigma^{\prime}, \gamma \sigma^{\prime \prime}}\left(\mu_{\gamma}\right) \mathcal{V}_{c s^{\prime \prime}, a s}^{\gamma \sigma^{\prime \prime}, \alpha \sigma}\left(\mu_{\gamma}+\varepsilon_{c s^{\prime \prime}}-\varepsilon_{a s}\right) \\
& -\Theta_{\omega-\varepsilon_{b s^{\prime}}+\varepsilon_{c s^{\prime \prime}}-\mu_{\gamma}} \mathcal{V}_{b s^{\prime}, c s^{\prime \prime}}^{\gamma \sigma^{\prime \prime}, \alpha \sigma}\left(\mu_{\gamma}-\varepsilon_{c s^{\prime \prime}}+\varepsilon_{b s^{\prime}}\right) \mathcal{V}_{c s^{\prime \prime}, a s}^{\beta \sigma^{\prime}, \gamma \sigma^{\prime \prime}}\left(\mu_{\gamma}\right),
\end{aligned}
$$

where $\Theta_{\omega}=\Theta(D-|\omega+i \Gamma|), D<D_{0}$ is the running cutoff and $\Gamma$ is the decoherence rate cutting off the RG flow [9]. Motivated by the expressions entering the computation of the current we use the mean value of the rates,
$\Gamma=\frac{1}{4} \sum_{a s} \Gamma_{a s}$. Generalising the rate (7) from PT using frequency-dependent coupling functions, we find

$$
\begin{aligned}
& \Gamma_{a s}\left(\varepsilon_{a s}\right)=\frac{1}{2} \int_{\mu_{\alpha}}^{\mu_{\beta}+\varepsilon_{a s}-\varepsilon_{b s^{\prime}}} \mathrm{d} \omega \frac{\left|N_{0} \mathcal{V}_{a s ; b s^{\prime}}^{\beta \sigma^{\prime} ; \alpha \sigma}(\omega)\right|^{2}}{\mu_{\beta}+\varepsilon_{a s}-\varepsilon_{b s^{\prime}}-\mu_{\alpha}} \\
& \times \int \mathrm{d} \omega^{\prime} A_{b s^{\prime}}\left(\omega^{\prime}\right) \int \mathrm{d} \omega^{\prime \prime}\left(1-f^{\alpha}\left(\omega^{\prime \prime}\right)\right) f^{\beta}\left(\omega^{\prime \prime}-\varepsilon_{a s}+\omega^{\prime}\right)
\end{aligned}
$$

with the Fermi function $f^{\alpha}(\omega)=1 /\left(1+e^{\left(\omega-\mu_{\alpha}\right) / T}\right)$. Here we have used that the spectral function $A_{a s}(\omega)$ is given by a Lorentzian of width $\Gamma$ centred around $\omega=\varepsilon_{a s}$ (replacing the $\delta$-function) and thus is strongly peaked. The convolution of the Fermi functions $i$ with broadened spectral functions leads to an effective temperature cutting off $i$ the flow to strong coupling at low bias voltages 15]. The equations (8) and (9) have to be solved self-consistently; we find a good convergence within a few iterations.

Typical results for the flow of the frequency-dependent Kondo couplings $J$ are shown in fig. 2, where we focus on one representative component of $J^{z, \perp}$ and $P^{0, x}$. In order to assess the importance of the orbital PS contributions, a comparison between results with and without orbital PS is provided. Lowering the cutoff down to the selfconsistently determined rate $\Gamma$ leads to an overall increase of $J$ with respect to the bare initial couplings $J_{0}$. Furthermore, pronounced peaks develop in correspondence of resonant transport involving spin- and orbital-flip processes. These peaks are split due to the finite bias by $\pm V / 2$. The singular behaviour is cut off by $\Gamma$; the induced noise due to the stationary current leads to a finite life time of the dot states 9]. This behaviour as well as the position of the resonances can be inferred from the analytic structure of the flow equations. In the following we focus on the coupling $J^{z}$, the RG equations of the other couplings can be found in ref. [17]. Explicitly, the RG equation for one representative component of $J^{z}$ in the absence of orbital PS reads

$$
\begin{aligned}
& \frac{\partial\left(J_{\uparrow}^{z}\right)_{11}^{L R}(\omega)}{\partial \ln D}= \\
& -\frac{1}{4}\left[\left(J_{\uparrow}^{\perp}\right)_{11}^{\gamma R}\left(\mu_{\gamma}+B\right)\left(J_{\downarrow}^{\perp}\right)_{11}^{L \gamma}\left(\mu_{\gamma}\right) \Theta_{\omega-\mu_{\gamma}-B}\right. \\
& \quad+\left(J_{\uparrow}^{\perp}\right)_{11}^{L \gamma}\left(\mu_{\gamma}\right)\left(J_{\downarrow}^{\perp}\right)_{11}^{\gamma R}\left(\mu_{\gamma}-B\right) \Theta_{\omega-\mu_{\gamma}+B} \\
& \quad+\left(J_{\uparrow}^{\perp}\right)_{21}^{\gamma R}\left(\mu_{\gamma}+\delta+B\right)\left(J_{\downarrow}^{\perp}\right)_{12}^{L \gamma}\left(\mu_{\gamma}\right) \Theta_{\omega-\mu_{\gamma}-\delta-B} \\
& \left.\quad+\left(J_{\uparrow}^{\perp}\right)_{21}^{L \gamma}\left(\mu_{\gamma}\right)\left(J_{\downarrow}^{\perp}\right)_{12}^{\gamma R}\left(\mu_{\gamma}-\delta-B\right) \Theta_{\omega-\mu_{\gamma}+\delta+B}\right] .
\end{aligned}
$$

Characteristic Kondo divergences appear at $\omega= \pm(\delta+B)$ and $\omega= \pm B$, which are split by $\pm V / 2$ in presence of a finite bias voltage. Similarly to the conventional Kondo model, $J^{z}$ is renormalised by two spin-flip processes $\propto\left(J^{\perp}\right)^{2}$. Schematically, the RG equation has the form $\partial J /(\partial \ln D)=-2 J^{2}$ thus leading to a divergence at low energies. 


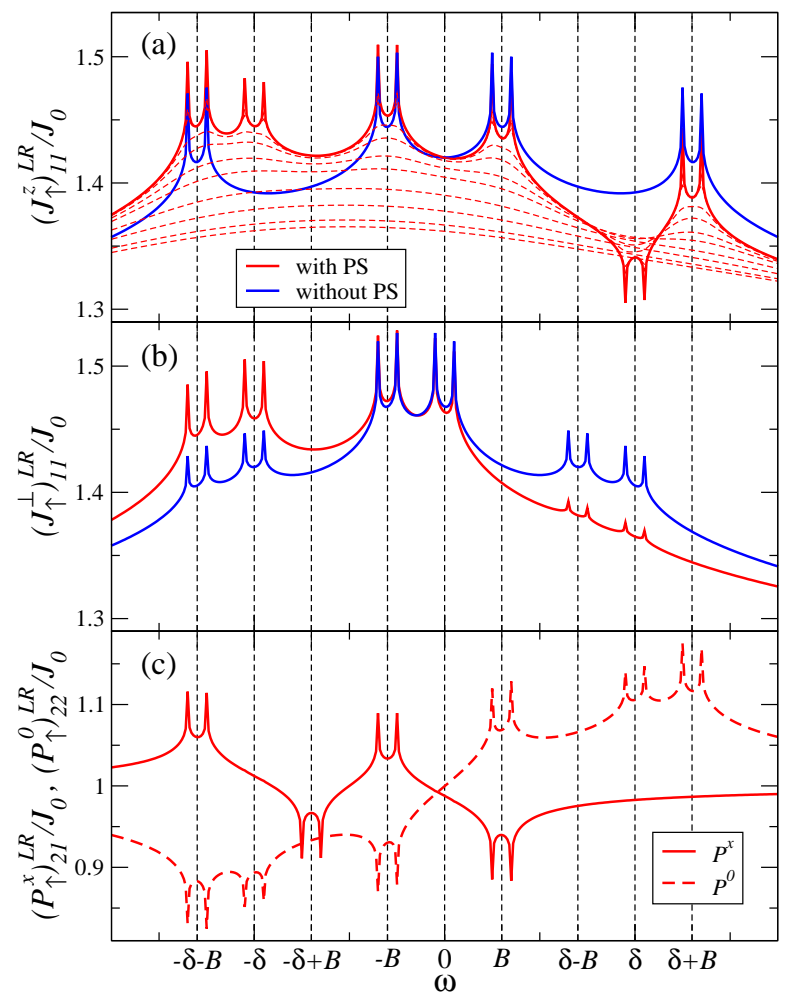

FIG. 2: (Colour online) Flow of the couplings $J^{z}$ (a), $J^{\perp}$ (b) and $P^{0, x}$ (c) as a function of $\omega$ for $r=1, B=0.3$, $T=0.01$, and $V=0.1$; the initial values are $N_{0} J_{0}=0.01$ and $D_{0}=1000$. Results with (red) and without (blue) orbital PS are compared. The evolution of $J^{z}$ under the RG flow is indicated by dashed red lines; the final value is obtained at $D=\Gamma \approx 0.0002$. We note that the $\mathrm{RG}$ flow results in $J^{z} \neq J^{\perp}$ and that the orbital PS yields new structures in $J^{z}$ at $\omega=-\delta \pm V / 2$ and $\omega=\delta \pm V / 2$. The orbital PS is only weakly renormalised.

Qualitatively new effects arise in the presence of orbital PS contributions, which lead to the following additional terms on the right-hand side of (10)

$$
\begin{aligned}
& -\frac{1}{4}\left(J_{\uparrow}^{z}\right)_{21}^{L \gamma}\left(\mu_{\gamma}\right)\left(P_{\uparrow}^{x}\right)_{12}^{\gamma R}\left(\mu_{\gamma}-\delta\right) \Theta_{\omega-\mu_{\gamma}+\delta} \\
& +\frac{1}{4}\left(J_{\uparrow}^{z}\right)_{21}^{\gamma R}\left(\mu_{\gamma}+\delta\right)\left(P_{\uparrow}^{x}\right)_{12}^{L \gamma}\left(\mu_{\gamma}\right) \Theta_{\omega-\mu_{\gamma}-\delta}+\left\{J^{z} \leftrightarrow P^{x}\right\} .
\end{aligned}
$$

$P^{x}$ always involves a change of the orbital index with energy $\pm \delta$. As can be seen in fig. 2 the orbital PS couplings are only weakly renormalised. For the symmetric case the orbital PS couplings $P$ do not flow at lowest order, they only evolve due to the finite frequency-dependence acquired by the Kondo couplings during the flow. Moreover, the averages of $P^{x}$ entering the expressions for the rates (9) and the current (12) will only slightly differ from the bare values and thus induce only minor effects. However, the flow of $P$ feeds back into the flow of the Kondo couplings where it results in qualitatively new features.
As shown in fig. 2a, for $\omega=\delta \pm V / 2$ the flow of $J^{z}(\omega)$ exhibits a crossover from a gradual increase to a dip as $\Gamma$ decreases. In this regime the flow equation assumes the form $\partial J /(\partial \ln D)=J P / 2$; the reversed sign with respect to the characteristic Kondo divergence gives rise to the observed dips. These are not present for $B=0$ as they merge with the larger peak coming from $\delta+B$. In this case the orbital PS terms only induce an asymmetry as a function of $\omega$. This can be observed for $J^{\perp}$ (see fig. 2b), where the contribution of the orbital PS only changes the weight of already resonant processes. $J^{\perp}$ is renormalised by $J^{\perp} J^{z}$ and $J^{\perp} P$, where both $J^{z}$ and $P$ do not involve spin-flip processes and thus no difference appears at finite magnetic field. For asymmetric couplings $r>1$ we find that existing peaks at $\omega=0, \pm B$ are enhanced while all others are strongly suppressed.

\section{CURRENT AND DOT OCCUPATION}

The current is obtained by taking into account the generated frequency dependence of the couplings. Specifically we find

$$
\begin{aligned}
I= & \frac{e}{2 \hbar} \int_{-\varepsilon_{b s^{\prime}}+\varepsilon_{a s}+\mu_{R}}^{\mu_{L}} \mathrm{~d} \omega \frac{\left|N_{0} \mathcal{V}_{a s ; b s^{\prime}}^{R \sigma ; L \sigma^{\prime}}(\omega)\right|^{2}}{\mu_{L}+\varepsilon_{b s^{\prime}}-\varepsilon_{a s}-\mu_{R}} \\
& \times \int \mathrm{d} \omega^{\prime} \mathcal{A}\left(\omega^{\prime}\right) \int \mathrm{d} \omega^{\prime \prime}\left[n_{b s^{\prime}}\left(1-f^{R}\left(\omega^{\prime}+\omega^{\prime \prime}\right)\right) f^{L}\left(\omega^{\prime \prime}\right)\right. \\
& \left.+n_{a s} f^{R}\left(\omega^{\prime}+\omega^{\prime \prime}\right)\left(1-f^{L}\left(\omega^{\prime \prime}\right)\right)\right] \\
& +\{\text { same expression with } L \leftrightarrow R\},
\end{aligned}
$$

with $\mathcal{A}(\omega)$ a Lorentzian [19] of width $2 \Gamma$ centred around $\varepsilon_{b s^{\prime}}-\varepsilon_{a s}$. Given the complicated form of the RG equations a detailed analytical understanding of the behaviour of the physical observables is not feasible, especially as weighted integrals over the coupling functions are involved. Thus we have evaluated the dot occupation numbers, the decoherence rates and the current numerically. The renormalisation of the energy levels and the magnetic field are neglected in the present analysis, since they turn out to contribute only at higher order.

First we present results for the symmetric situation $r=1$ and discuss the effects of a finite magnetic field. Fig. 3a shows the differential conductance obtained from PT and RG calculations both with and without orbital PS. As long as the couplings are symmetric, the differential conductance is symmetric with respect to the voltage and thus only $V>0$ is shown. We observe that the inclusion of the orbital PS leads to an enhancement of the conductance, which is simply due to the increase of transport channels. This enhancement is voltage independent except that its value changes at $V=\delta$, which indicates the importance of the coupling of the orbital PS terms to the $J^{z}$ processes. Regarding the effect of the $\mathrm{RG}$ we observe an overall increase of the conductance, which is due to the increase of the Kondo couplings under the RG 


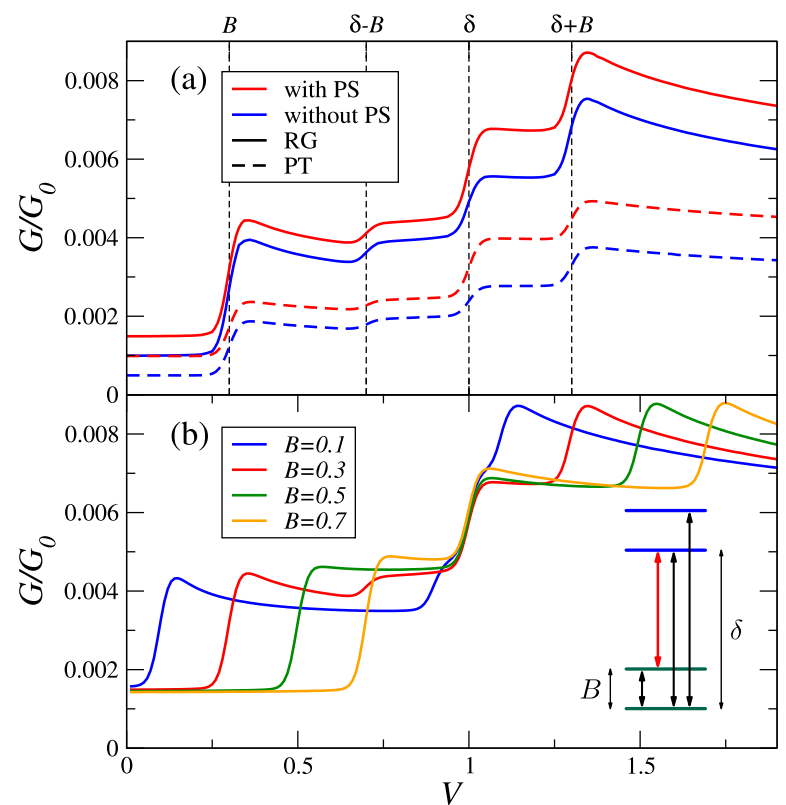

FIG. 3: (Colour online) Differential conductance, $G=\mathrm{d} I / \mathrm{d} V$, normalised to $G_{0}=2 e^{2} / h$, for symmetric couplings $(r=1)$; a) RG results (full lines) are compared to PT (dashed lines), both with (red) and without (blue) orbital PS for parameters as in fig. 2. b) RG results including orbital PS for the same parameters but different values of $B$. The cascade step is visible at $V=\delta-B$ provided $B<\delta / 2$; Inset: schematic representation of the possible transitions between the dot orbitals, the cascade transition being marked in red.

flow (see fig. 22). Furthermore, there is a clear enhancement of the inelastic cotunneling signatures at $V=B$ and $\delta+B$ involving spin-flip processes. In contrast, the effect of the orbital PS remains unchanged under the RG flow.

The cascade step [12] at $V=\delta-B$ has its physical origin in the non-equilibrium dot occupations (see fig. $4 \mathrm{a}$ ). For $V>B$ the orbital $1 \downarrow$ gets populated from which the next higher orbital $2 \uparrow$ is split by $\delta-B$ (see inset of fig. 3b). Therefore, a finite population of $2 \uparrow$ already sets in at $V=\delta-B$, which results in the cascade step in the conductance displayed in fig. 3. This is in analogy to the Balmer series observed in the traditional spectroscopy of hydrogen, where instead of transitions from the ground state (Lyman series) the transitions from an excited state are probed. As one can observe in fig. [3a the cascade resonance is only weakly enhanced by RG. The reason might be that the RG only starts to play a role at higher orders since the cascade effect is due to transitions from an excited state. Clearly the cascade effect is not present at $B=0$ while for $B=\delta / 2$ it is superimposed by the inelastic cotunneling process at $V=B$ (see fig. $3 \mathrm{~b}$ ).

In the following we discuss the effect of asymmetric couplings and the resulting NDC. Fig. 4 shows PT and $\mathrm{RG}$ results for the dot occupations and differential conductance for asymmetric couplings with $r=5$. This

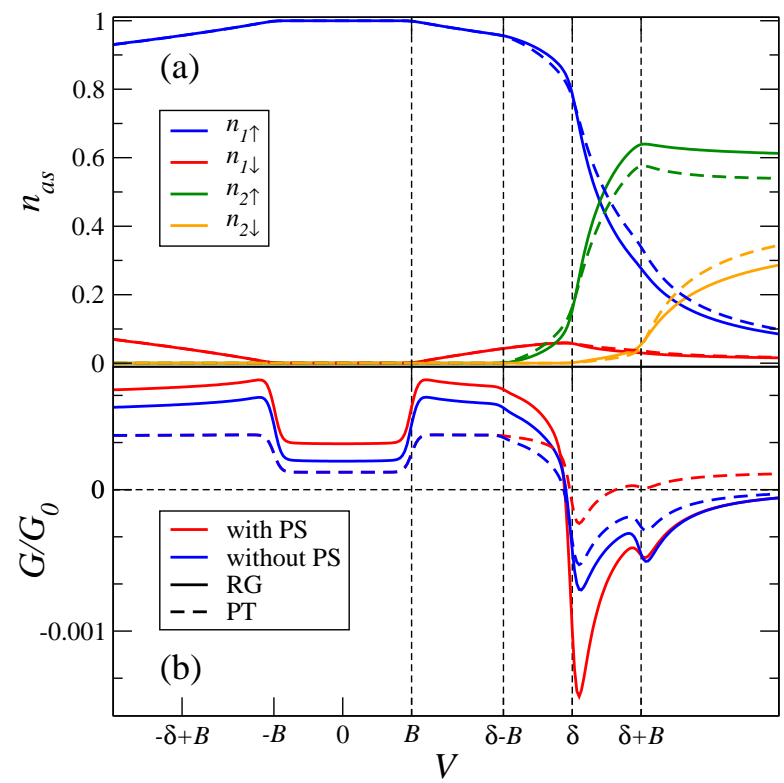

FIG. 4: (Colour online) Dot occupation numbers (a) and differential conductance (b) for asymmetric couplings with $r=5, N_{0} J_{0}=0.005$ and other parameters as in fig. 2. For the occupation numbers RG results (full lines) are compared to PT (dashed lines) both including orbital PS.

implies that $t_{R 1} \gg t_{R 2}$, i.e. the transport through the second orbital is strongly suppressed. At $V \approx \delta$ we observe a strong increase of $n_{2 \uparrow}$ at the cost of $n_{1 \uparrow}$ which consequently leads to a decrease in the current and thus the appearance of NDC. The increase of $n_{2 \downarrow}$ similarly yields a side peak located at $V \approx \delta+B$. A simple explanation for the appearance of the NDC is provided by PT in the limit of $r \gg 1, B=0$ and $T=0$. Introducing the orbital polarisation $M_{12}=\frac{1}{2} \sum_{\sigma}\left(n_{1 \sigma}-n_{2 \sigma}\right)$ we obtain for the differential conductance at $V \approx \delta$

$$
\frac{G}{G_{0}} \propto 2+3 M_{12}+\left.\delta \frac{\mathrm{d} M_{12}}{\mathrm{~d} V}\right|_{V=\delta} .
$$

This shows that the NDC originates in the voltagedependent occupation numbers and that a sufficiently strong occupation inversion $\left(M_{12}<0\right)$ and/or sufficiently fast reduction of the orbital polarisation $\left(\mathrm{d} M_{12} / \mathrm{d} V<0\right)$ are necessary. We note that the second orbital is not occupied for negative voltages, thus NDC is absent in this regime. For generic $B$ and $r$ NDC shows up in the vicinity of $n_{1 \uparrow}=n_{2 \uparrow}$ in the interval $\delta<V<\delta+B$. NDC is favoured for small magnetic fields, as the occupation inversion becomes less feasible with increasing Zeeman splitting. While the dot occupations do not exhibit remarkable renormalisation effects with respect to the PT

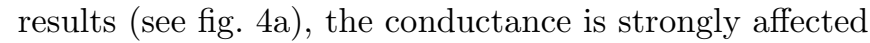
by both renormalisation effects and orbital PS contributions. The NDC is strongly enhanced by the RG, and in contrast to the case of symmetric couplings (see fig. 3a ) the orbital PS has opposite effects on the PT and RG results: while the orbital PS reduces the NDC in PT, 
it induces an increase in the $\mathrm{RG}$ results. Depending on the initial system parameters the NDC might even exclusively be generated during the RG flow. At low bias voltage the effect of the orbital PS contributions is strongly reduced in $\mathrm{PT}$, from the analytic calculations this effect can be shown to be proportional to $\left(P^{0}\right)^{2} \sim 1 / r^{2}$. In contrast, the corresponding RG results show a marked $r$ and $V$-dependence of the orbital PS contributions. Concerning the cascade effect the onset of $n_{2 \uparrow}$ becomes more pronounced with stronger asymmetry.

We stress that the mechanism leading to NDC in the present model is fundamentally different from the NDC observed in the interacting resonant level model [18] where it has its origin in a voltage-dependent renormalisation of the hopping amplitudes towards smaller values.

Finally we note that the temperature considered here is $T=0.01 \delta \gg T_{K}$ with the rough estimate $T_{K} \sim$ $D_{0} \exp \left[-1 /\left(N_{0} J_{0}\right)\right]$. For higher temperatures $T \sim 0.1 \delta$ thermal smearing inhibits a clear distinction of the different transitions. The small absolute values of the conductance are due to the small values of the initial couplings $N_{0} J_{0} \sim 0.01$ required by our perturbative treatment. However, we expect the cascade effect as well as the appearance of NDC to be generic features of non-equilibrium transport through multi-orbital quantum dots. In particular, these features are expected to show up in the kind of transport experiments on molecules and nanowires as carried out in Refs. [27] where the couplings to the leads will generically be strongly asymmetric and orbital dependent.

\section{CONCLUSION}

We studied a minimal model for a multi-orbital quantum dot exhibiting the cascade effect and NDC, namely a two-orbital Anderson model with asymmetric and orbital-dependent hopping amplitudes in a magnetic field. We considered the regime of strong Coulomb interactions where a SW transformation leads to an effective Kondo model including orbital PS terms. The non-equilibrium transport through this system was studied using a perturbative RG treatment with frequencydependent couplings. In particular, the RG flow leads to a strong enhancement of the NDC. The observed strong influence of the orbital PS on the scaling of the Kondo couplings indicates an interaction-driven interplay of spin and orbital degrees of freedom even in absence of spin-orbit interactions or spin-dependent tunneling amplitudes. The inclusion of additional spin-orbit interactions represents an interesting extension beyond the scope of the present analysis.

We are grateful to K. Flensberg, S. Jakobs, T. S. Jespersen, J. Nygård, J. Paaske, and H. Schoeller for helpful discussions. We acknowledge kind hospitality at the Niels Bohr Institute, University of Copenhagen (S.A. and S.M.) and the Department of Physics, University of Basel (S.M.). This work was supported by the DFG-FG 723 and 912, and the Robert Bosch Foundation.
[1] Hanson R. et al., Rev. Mod. Phys. 79, 1217 (2007).

[2] Chen J. et al., Science 286, 1550 (1999).

[3] Osorio E. A. et al., Nano Lett. 7, 3336 (2007).

[4] Roch N. et al., Nature 453, 633 (2008).

[5] Nygård J. et al., Nature 408, 342 (2000); Sapmaz S. et al., Semicond. Sci. Technol. 21, S52 (2006).

[6] Paaske J. et al., Nat. Phys. 2, 460 (2006).

[7] Jespersen T. S. et al., Phys. Rev. B 74, 233304 (2006); Csonka S. et al., Nano Lett. 8, 3932 (2008).

[8] See, for example, Andergassen S. et al., Nanotechnology 21, 272001 (2010) and references therein.

[9] Rosch A. et al., Phys. Rev. Lett. 90, 076804 (2003); J. Phys. Soc. Jpn. 74, 118 (2005).

[10] Kehrein S., Phys. Rev. Lett. 95, 056602 (2005); Anders F. B., Phys. Rev. Lett. 101, 066804 (2008); Jakobs S. G., Pletyukhov M. and Schoeller H., Phys. Rev. B 81, 195109 (2010); Eckel J. et al., New J. Phys. 12, 043042 (2010).

[11] Schoeller H., Eur. Phys. J. Special Topics 168, 179
(2009)

[12] Schmaus S. et al., Phys. Rev. B 79, 045105 (2009).

[13] Schrieffer J. R. and Wolff P. A., Phys. Rev. 149, 491 (1966).

[14] Yang Y.-F. and Held K., Phys. Rev. B 72, 235308 (2005).

[15] Paaske J., Rosch A. and Wölfle P., Phys. Rev. B 69, 155330 (2004); Paaske J. et al., Phys. Rev. B 70, 155301 (2004).

[16] Abrikosov A. A., Physics 2, 61 (1965); Coleman P., Phys. Rev. B 29, 3035 (1984).

[17] Müller S., Diploma thesis, RWTH Aachen 2010.

[18] Doyon B., Phys. Rev. Lett. 99, 076806 (2007); Boulat E., Saleur H. and Schmitteckert P., Phys. Rev. Lett. 101, 140601 (2008); Borda L. and Zawadowski A., Phys. Rev. B 81, 153303 (2010); Karrasch C. et al., Europhys. Lett. 90, 30003 (2010).

[19] $\mathcal{A}(\omega)$ is given by the convolution of $A_{a s}(\omega)$ and $A_{b s^{\prime}}(\omega)$. 